Extracellular matrix formation (ECM) and remodeling are critical events related to the pathogenesis of pulmonary fibrosis. Matrix metalloproteinases play an essential role in degrading and remodeling the ECM. In this study, we tried to show the presence and correlation of promatrix metalloproteinase-3 (proMMP-3) (the inactive form of metalloproteinase3) levels in coal workers' pneumoconiosis (CWP) with different categories.

The study population consisted of 44 coal miners with CWP (pos CWP). Coal miners without CWP (neg CWP, $n=24$ ) and non-underground personnel (controls, $n=17$ ) were taken as controls. All coal miners were stable and had no systemic infection or disease. Standard posterio-anterior chest radiographs and pulmonary function tests were performed to exclude any diseases other than CWP. Serum proMMP-3 was analysed using the sandwich enzyme-linked immunosorbent assay according to the manufacturer's instructions (The Binding Site, Birmingham, UK).

Mean proMMP-3 values of the all three groups were compared and a significant statistical difference obtained $(p<0.001)$. In addition, a statistically significant difference was found between categories of the disease and proMMP-3 values $(p<0.05)$. The effects of age, exposure duration and cigarette smoking on proMMP-3 values in coal miners with CWP were investigated. There were no correlations between age, smoking and proMMP-3 values. However, a positive correlation was found between exposure duration and proMMP- 3 values $(r=0.447, p=0.008)$. In conclusion, proMMP-3 (prostromelysin 1) may play an essential role in degrading and remodeling the ECM in workers with pneumoconiosis. ProMMP-3 may also reflect the stage of pneumoconiosis disease.

\section{The presence of promatrix metalloproteinase- 3 and its relation with different categories of coal workers' pneumoconiosis}

\author{
Remzi Altin ${ }^{1, \mathrm{CA}}$, Levent Kart ${ }^{1}$, İshak Tekin ${ }^{2}$, \\ Ferah Armutcu ${ }^{3}$, Meltem Tor $^{1}$ and Tacettin Örnek ${ }^{1}$
}

${ }^{1}$ Department of Pulmonary Medicine, ${ }^{2}$ Department of Immunology and ${ }^{3}$ Department of Biochemistry, Faculty of Medicine, Zonguldak Karaelmas University, 67600 Zonguldak, Turkey

\footnotetext{
${ }^{\mathrm{CA}}$ Corresponding author

Tel: +90372 2610169

Fax: +90372 2610155

E-mail: remal1@yahoo.com
}

\section{Introduction}

Matrix metalloproteinases (MMPs) are a member of a group of enzymes that can break down proteins, such as collagen, that are normally found in the spaces between cells in tissues (i.e. extracellular matrix proteins). Extracellular matrix degradation involves the MMPs, a conserved family with a zinc binding site in the catalytic domain, and an amino terminal domain responsible for the zymogen inactive state. The MMP family includes: the collagenases, which degrade fibrillar collagens; the stromelysins, which cleave proteoglycans and some glycoproteins; the gelatinases $\mathrm{A}$ and $\mathrm{B}$, which degrade basement membrane type IV collagen; and the membrane-type metalloproteinases, which are able to activate progelatinase A. ${ }^{1,2}$ MMP activity is regulated at different levels including the transcriptional level, the proenzyme activation, and the inhibition of active enzymes by a family of tissue inhibitors of metalloproteinases. ${ }^{3-5}$

MMPs play a central role in many biological processes, such as embryogenesis, normal tissue remodeling, wound healing, and angiogenesis, and in diseases such as atheroma, arthritis, cancer, and tissue ulceration. ${ }^{6}$ MMPs might present inflammatory disease activity. ${ }^{7-10}$

MMP-3 (stromelysin 1) is an enzyme with a broad substrate specifity and it is capable of degrading connective tissue components such as proteoglycan, laminin fibronectin, collagen IV and other denatured collagens. In addition to matrix degradation, it may activate other MMPs such as collagenase. ${ }^{1,2}$ Promatrix metalloproteinase-3 (proMMP-3) is the inactive form of metalloproteinase- 3 . The advantage of proMMP-3 may be the higher stability and longer half-life in the circulation, as its affinity to inhibitor proteins, such as tissue inhibitors of metalloproteinase, may be much less than that of the active form, 
saving proMMP-3 from early elimination out of serum. $^{11,12}$

Coal workers' pneumoconiosis (CWP) is the parenchymal lung disease that results from the inhalation and deposition of coal mine dust and the tissue's reaction to its presence. ${ }^{13}$ The course of CWP is often insidious, and progression occurs in the absence of continued exposure to coal, leading to chronic respiratory insufficiency and even death. Chronic exposure to coal particles results in macrophagelymphocytic granulomatous lung inflammation, which is followed by abnormal and progressive accumulation of the extracellular matrix. ${ }^{14}$ However, the pathogenic mechanisms and the sequence of the pathological events leading to the fibrotic response have not been well defined.

To the best of our knowledge, there are not enough studies to show the role of MMPs in coal miners with pneumoconiosis in the literature. Therefore, in this study we aimed to show the presence of proMMP-3 (prostromelysin 1) and its relation with different categories of CWP (disease severity).

\section{Materials and methods}

The study population consisted of coal miners with and without CWP and non-underground personnel evaluated between June 2002 and August 2002 at the Zonguldak Karaelmas University Medical Faculty Hospital. Subjects worked at least 8 years in Zonguldak Coal Basin. All coal miners were men and working in the coal production unit. There were 44 coal miners with positive CWP (pos CWP). Twentyfour coal miners who were CWP negative (neg CWP) and 17 non-underground personnel (controls) who were not exposed to any toxic agents (coal dust, gases, etc.) were taken as control groups. All coal miners were stable and had no systemic infection or disease. Patients with previous diagnosis of COPD, asthma and any pulmonary disease were also excluded. Pulmonary function tests were assessed in all patients to exclude any obstructive disease that was not previously diagnosed. A chest physician filled in a questionnaire to obtain clinical symptoms, demographic data and smoking histories for every study subject.

Informed written consent was obtained from all subjects.

\section{Chest radiographs}

Chest radiographs were obtained for all coal miners with or without a prior, confirmed diagnosis of CWP. The chest radiographs were graded by a B-type trained reader who had a good knowledge of the International Labour Office classification criteria. ${ }^{15}$
The International Labour Office scoring system is based on the reader's assessment of small opacities compared with a set of standard radiographs. After grading, category 0 (profusions $0 /-$ to $0 / 1$ ), category 1 (profusions $1 / 0$ to $1 / 2$ ), category 2 (profusions $2 / 1$ to $2 / 3$ ) and category 3 (profusions $3 / 2$ only) were included. No coal miners with major opacity were enrolled in the study due to an insufficient number of cases.

\section{Enzyme-linked immunosorbent assay}

Sera were prepared after blood collection and all samples were stored at $-20^{\circ} \mathrm{C}$ before analysis. The proMMP-3 level was analysed using the sandwich enzyme-linked immunosorbent assay for serum proMMP-3 according to the manufacturer's instructions (The Binding Site, Birmingham, UK).

\section{Statistical analysis}

Statistical analysis was carried out by means of the SPSS software (version 11.0; SPSS Inc., USA). Data are presented as the median value and the lower (25\%) and upper (75\%) quartiles. The differences between groups were analysed by the Mann-Whitney U-test. The difference between the means of variables in more than two groups was evaluated by the KruskalWallis variance analysis test. The relations between proMMP-3 values and age, exposure duration and smoking were examined by the Pearson rank correlation coefficient. Significance was set at the 5\% level $(p<0.05)$. All measured values are presented as mean \pm standard deviation.

\section{Results}

Demographic characteristics and mean proMMP-3 values of coal miners with CWP (pos CWP), without CWP (neg CWP) and non-underground personnel (controls) are presented in Table 1. A mean proMMP3 value of the groups is also shown in Fig. 1. Considering demographic characteristics, there was no statistical difference between groups concerning age $(p>0.05)$. Working durations for the pos CWP and neg CWP were similar and no statistical difference was found $(p>0.05)$. Mean proMMP-3 values of the all three groups were compared and a significant statistical difference obtained $(p<0.001)$.

The effects of age, exposure duration and cigarette smoking on proMMP-3 values in coal miners with CWP were investigated. There was no correlation between age and proMMP-3 values $(r=0.196$, $p=0.266)$. No correlation was also obtained between smoking and proMMP-3 values ( $r=0.229, p=0.113$ ). However, a positive correlation was found between 
Table 1. Comparisons of demographic characteristics and proMMP-3 values of coal miners with pneumoconiosis (pos CWP) and without pneumoconiosis (neg CWP), and of non-underground personnel (controls)

\begin{tabular}{lllll}
\hline & Pos CWP $(n=44)$ & Neg CWP $(n=24)$ & Controls $(n=17)$ & $p$ value \\
\hline Age (years) & $41.0 \pm 3.9(36-47)$ & $37.8 \pm 4.9(29-43)$ & $34.9 \pm 3.7(27-39)$ & $>0.05$ \\
Duration of coal exposure (years) & $15.8 \pm 4.0(9-22)$ & $13.3 \pm 3.4(8-17)$ & None & $>0.05$ \\
Smoker ratio & $30 / 44(68.1 \%)$ & $15 / 24(62.5 \%)$ & $11 / 17(64.7 \%)$ & $>0.05$ \\
Smoking (packets/year)* & $18.9 \pm 10 .(6-45)$ & $16.6 \pm 4.7(10-22)$ & $16.8 \pm 5.4(10-24)$ & $>0.05$ \\
Metalloproteinase-3 $(\mathrm{ng} / \mathrm{ml})$ & $114 \pm 92^{\dagger}(13-575)$ & $53 \pm 31^{\ddagger}(8-98)$ & $44 \pm 34^{\text {NS }}(2-112)$ & $<0.001$ \\
\hline
\end{tabular}

Data presented as median \pm standard deviation (range), except smoker ratio.

* Cumulative mean values only for those who smoke in each group.

${ }^{\dagger}$ Comparison of pos CWP and controls, $p>0.001 ;{ }^{\star}$ comparison of pos CWP and neg CWP, $p<0.01$; ${ }^{*}$ not significant, comparison of neg CWP and controls, $p>0.05$.

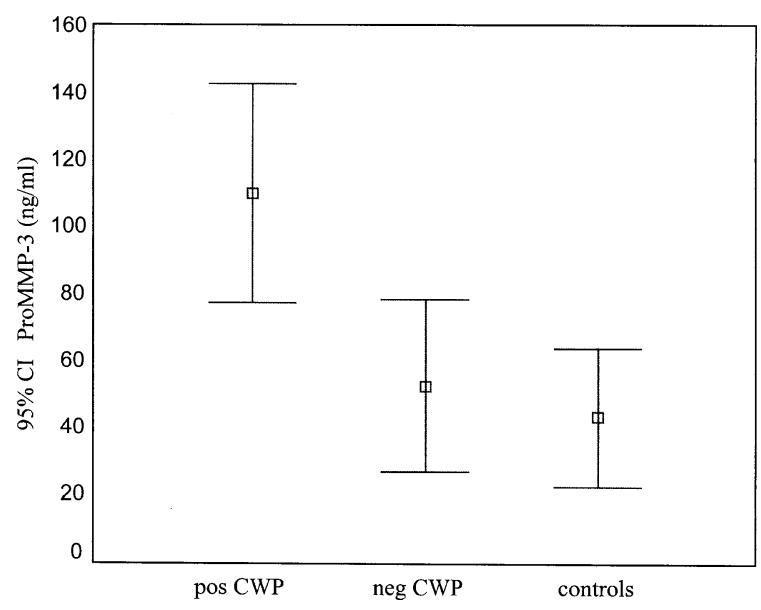

FIG. 1. Metalloproteinase-3 values of coal miners with pneumoconiosis (pos CWP) and without pneumoconiosis (neg CWP), and of non-underground personnel (controls). $95 \% \mathrm{Cl}, 95 \%$ confidence interval.

exposure duration and proMMP-3 values $(r=0.447$, $p=0.008$; Fig. 2).

Patients' mean proMMP-3 values according to their profusional categories are presented in Table 2, and statistically significant differences obtained between categories of the disease and proMMP-3 values.

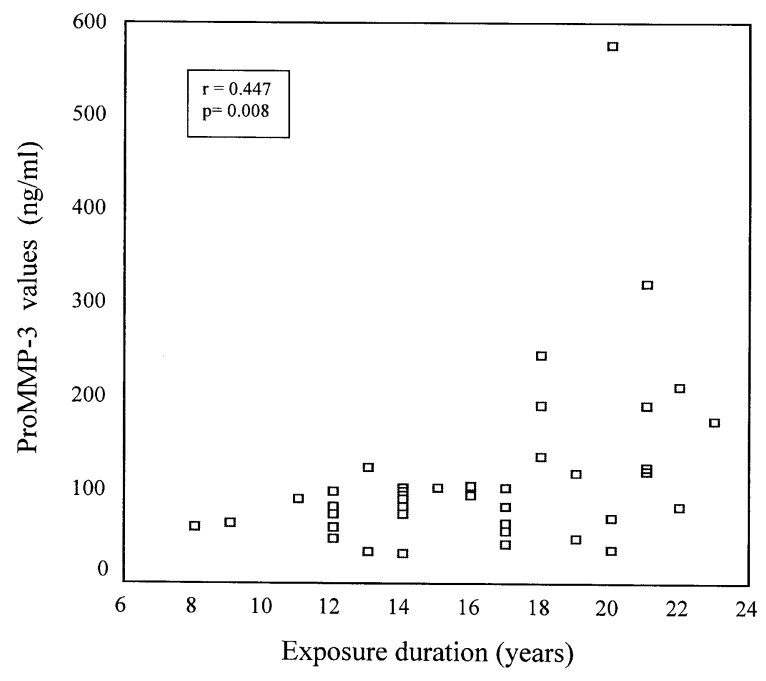

FIG. 2. Correlation between proMMP-3 values and exposure duration in coal miners with pneumoconiosis.

\section{Discussion}

Chronic exposure to coal particles results in macrophage-lymphocytic granulomatous lung inflammation, which is followed by abnormal and progressive accumulation of the extracellular matrix. ${ }^{14,16}$ It is clear that we have the need to further our basic and clinical science knowledge of this complex process. Additionally, we lack information regarding the natural history of pulmonary fibrosis in CWP, and we are left with only descriptive 'snapshots' of the histopathology.

Pulmonary lesions in CWP may be an example of dysregulated inflammation, repair and remodeling of the lung in response to continuous coal dust exposure in susceptible workers. In contrast to normal repair, the persistence of chronic inflammation ${ }^{17}$ in CWP promotes fibroproliferation and deposition of the extracellular matrix, reflecting dysregulated and exaggerated tissue repair. The studies carried out on CWP inflammation have shown that alveolar macrophages play a dominant role in inflammation and that alveolar macrophage recruitment is related to the expression and function of chemokines. Several cytokines participate in the local injury and inflammatory reaction. These are interleukin (IL)-1, IL-8, monocyte chemotactic protein-, tumor necrosis factor- $\alpha$, platelet-derived growth factor, insulin-like growth factor-1, transforming growth factor-beta,

Table 2. Comparison of mean metalloproteinase-3 values according to chest radiograph profusion categories

\begin{tabular}{lcc}
\hline $\begin{array}{c}\text { Chest radiograph } \\
\text { category }\end{array}$ & $\begin{array}{c}\text { Number of } \\
\text { patients }\end{array}$ & $\begin{array}{c}\text { Metalloproteinase-3 } \\
\text { values }(\mathrm{ng} / \mathrm{ml})\end{array}$ \\
\hline $\begin{array}{c}\text { Category } 0^{*} \\
\text { (p } 0 / 0 \text { and } 0 / 1)\end{array}$ & 24 & $53 \pm 31(8-98)$ \\
$\begin{array}{c}\text { Category } 1^{* *} \\
(\mathrm{p} 1 / 0 \text { to } 1 / 2)\end{array}$ & 28 & $73 \pm 26^{\mathrm{NS}}(13-118)$ \\
$\begin{array}{c}\text { Category } 2^{* * *} \\
\quad(\mathrm{p} 2 / 1 \text { to } 2 / 3)\end{array}$ & 11 & $129 \pm 134^{\dagger}(34-575)$ \\
$\begin{array}{c}\text { Category } 3^{* * *} \\
(\mathrm{p} 3 / 2 \text { only) }\end{array}$ & 5 & $176 \pm 73^{*}(91-322)$ \\
$p$ value & & $<0.01$ \\
\hline
\end{tabular}

Data presented as median + standard deviation (range).

* Coal miners without or suspected CWP, ** early CWP, *** lowgrade CWP.

NS Not significant, comparison of category 0 and category 1 $p>0.05 ;{ }^{\dagger}$ Comparison of category 1 and category $2, p<0.05$; comparison of category 2 and category $3, p<0.05$. 
and basic-fibroblast growth factor. ${ }^{16,18}$ Tumor necrosis factor and IL-1 are important early response cytokines that are involved in fibroblast activation. In addition, the biology of recruitment may be regulated by other factors, such as metalloproteinases, that not only regulate extracellular matrix formation, but may regulate the specific receptors on these cell surfaces.

MMPs are a family of secreted or transmembrane zinc-dependent endopeptidases that are capable of degrading virtually all extracellular matrix and basement membranes components. ${ }^{2}$ Extracellular matrix formation and remodeling are critical events related to the pathogenesis of pulmonary fibrosis. MMPs play an essential role in degrading and remodeling the extracellular matrix.

In the literature, there are a few studies related with MMPs performed on coal miners with or without pneumoconiosis. In 1993, Porcher et al. investigated a metalloproteinase called plasma neutral metalloendo-peptidase elastase that was found to be elevated in the plasma of coal miners. ${ }^{19}$ Then in 1996, Soleilhac et al. described an endopeptidase as neutral endopeptidase $24.11 .^{20}$ In their study, they found an increase in serum neutral endopeptidase 24.11 activity of underground miners, compared with surface miners $(5.7$-fold increase, $p<0.01)$. The results were not correlated with systemic inflammation parameters, but seemed to reflect the chronic pulmonary inflammatory state induced by coalmine dust exposure, and so might be a marker of lung injury.

In the literature, there is no study to demonstrate the presence and affects of proMMP-3 (prostromely$\sin 1$ ) or its active form (metalloproteinase-3/stromelysin 1) in CWP. So in this study we aimed to show the presence of proMMP-3 and its relation with different categories of CWP (disease severity). We preferred proMMP-3 instead of the active form metalloproteinase-3. Because proMMP-3 has higher stability and longer half-life in the circulation, its affinity to inhibitor proteins, such as tissue inhibitors of metalloproteinase, may be much less than that of the active form, saving proMMP-3 from early elimination out of serum. ${ }^{11,12}$ The present study results showed a positive correlation between exposure duration and proMMP-3 values $(r=0.447$, $p=0.008$; Fig. 2), and a statistically significant difference obtained in comparison of mean proMMP-3 values of the all three groups defined as workers with pneumoconiosis, workers without pneumoconiosis and non-underground personnel $(p<0.001)$. No difference was obtained between workers without pneumoconiosis and non-underground personnel $(p>0.05)$. These results mean that proMMP-3 may play a role in degrading and remodeling the extracellular matrix in workers with pneumoconiosis and increases as the stage of the CWP increase.

Aging is an important factor for the MMP serum levels. According to the studies carried out in different illnesses rather than CWP, serum levels of MMPs increase with age. ${ }^{21-23}$ In our study, we investigated the effects of age on proMMP-3 values in coal miners with pneumoconiosis, but there was no correlation between age and proMMP-3 values ( $r=0.196, p=0.266$ ). This may be explained by the younger age of our population. In the literature, it is well known that smoking increase both tissue inhibitors of metalloproteinases and MMP levels. ${ }^{24,25}$ In the present study, no correlation was obtained between smoking and proMMP-3 values $(r=0.229$, $p=0.113$ ). We think that this may be due to the low number of study cases.

In conclusion, proMMP-3 (prostromelysin 1) may play a role in degrading and remodeling the extracellular matrix in workers with pneumoconiosis. ProMMP-3 may also reflect the stage of pneumoconiosis disease. To define exact roles of ProMMP-3 in coal workers with pneumoconiosis, further studies are required.

\section{References}

1. Birkedal-Hansen HW, Moore GI, Bodden MK, Windsor LJ, BirkedalHansen B, DeCarlo A, Engler JA. Matrix metalloproteinases: a review. Crit Oral Biol Med 1993; 4: 197-250.

2. Woessner JF. The family of matrix metalloproteinases. Ann NY Acad Sci 1994; 732: $11-21$

3. Gómez D, Alonso D, Yoshiji H, Thorgeirsson UP. Tissue inhibitors of metalloproteinases: structure, regulation and biological functions. Eur J Cell Biol 1997; 74: 111-122.

4. Shapiro SD, Senior RM. Matrix metalloproteinases: matrix degradation and more. Am Rev Respir Cell Mol Biol 1999; 20: 1100-1102.

5. Greenwald RA, Zucker S, Golub LM. Inhibition of matrix metalloproteinases: therapeutic implications. Ann NY Acad Sci 1999; 878: 761.

6. Visse R, Nagase H. Matrix metalloproteinases and tissue inhibitors of metalloproteinases: structure, function, and biochemistry. Circ Res 2003; 92: $827-839$

7. Katrib A, Tak PP, Bertouch JV, et al. Expression of chemokines and matrix metalloproteinases in early rheumatoid arthritis. Rheumatology 2001; 40: 988-994.

8. Posthumus MD, Limburg PC, Westra J, Cats HA, Stewart RE, van Leeuwen MA, van Rijswijk MH. Serum levels of matrix metalloproteinase-3 in relation to the development of radiological damage in patients with early rheumatoid arthritis. Rheumatology 1999; 38: 1081-1087.

9. Klimiuk PA, Sierakowski S, Latosiewicz R, Cylwik B, Skowronski J, Chwiecko J. Serum matrix metalloproteinases and tissue inhibitors of metalloproteinases in different histological variants of rheumatoid synovitis. Rheumatology 2002; 41: 78-87.

10. Arnaud C, Valérie LC, Frédérique N, et al. Stromelysin 1 (matrix metalloproteinase 3) and HLA-DRB1 gene polymorphisms: association with severity and progression of rheumatoid arthritis in a prospective study. Arthritis Rheumatism 2002; 46: 1754-1762.

11. Allan J, Hembry R, Angal S, Reynolds J, Murphy G. Binding of latent and high $\mathrm{Mr}$ active forms of stromelysin to collagen is mediated by the C-terminal domain. J Cell Sci 1991; 99: 789-795.

12. Suzuki K, Kan C, Hung W, Gehring M, Brew K, Nagase H. Expression of human pro-matrix metalloproteinase 3 that lacks the N-terminal 34 residues in Escherichia coli: autoactivation and interaction with tissue inhibitor of metalloproteinase 1 (TIMP-1). Biol Chem 1998; 379: 185191.

13. Lynn TT. Coal workers' lung disease and silicosis. In: Alfred PF, ed. Fishman's Manual of Pulmonary Diseases and Disorders, 3rd edn, McGraw-Hill, 2002: 238-249.

14. Rom WN. Basic mechanisms leading to focal emphysema in coal worker's pneumoconiosis. Environ Res 1990; 53: 16-28. 
15. International Labour Office (ILO). Guidelines for the use of ILO international classification of radiographs of pneumoconioses. Оссира tional Safety and Health Series, No. 48. Geneva: ILO, 1980.

16. Kuhn DC, Stauffer JL, Gaydos LJ, Demers LM. Inflammatory and fibrotic mediator release by alveolar macrophages from coal miners. $J$ Toxicol Environ Health 1995; 46: 9-21.

17. Brown GM, Brown DM, Donaldson K. Persistent inflammation and impaired chemotaxis of alveolar macrophages on cessation of dust exposure. Environ Health Perspect 1992; 97: $91-94$

18. Vaillant P, Menard O, Vignaud JM, Martinet N, Martinet Y. The role of cytokines in human lung fibrosis. Monaldi Arch Chest Dis 1996; 51 $145-152$.

19. Porcher JM, Lafuma C, el Nabout $\mathrm{R}$, et al. Biological markers as indicators of exposure and pneumoconiotic risk: prospective study. Int Arch Occup Environ Health 1993; 65: 209-213.

20. Soleilhac JM, Lafuma C, Porcher JM, Auburtin G, Roques BP. Characterization of a soluble form of neutral endopeptidase-24.11 (EC 3.4.24.11) in human serum: enhancement of its activity in serum of underground miners exposed to coal dust particles. Eur J Clin Invest 1996; 26: 1011 1017.

21. Manicourt DH, Fujimoto N, Obata K, Thonar EJ. Serum levels of collagenase, stromelysin-1, and TIMP-1. Age- and sex-related differences in normal subjects and relationship to the extent of joint involvement and serum levels of antigenic keratan sulfate in patients with osteoarthritis. Arthritis Rheum 1994; 37: 1774-1783

22. Ashcroft GS, Horan MA, Herrick SE, Tarnuzzer RW, Schultz GS, Ferguson MW. Age-related differences in the temporal and spatial regulation of matrix metalloproteinases (MMPs) in normal skin and acute cutaneous wounds of healthy humans. Cell Tissue Res 1997; 290: 581-591.

23. Guo L, Hussain AA, Limb GA, Marshall J. Age-dependent variation in metalloproteinase activity of isolated human Bruch's membrane and choroid. Invest Ophthalmol Vis Sci 1999; 40: 2676-2682.

24. Yin L, Morita A, Tsuji T. Alterations of extracellular matrix induced by tobacco smoke extract. Arch Dermatol Res 2000; 292: 188-194.

25. Liu PY, Chen JH, Li YH, Wu HL, Shi GY. Synergistic effect of stromelysin1 (matrix metallo-proteinase-3) promoter $5 \mathrm{~A} / 6 \mathrm{~A}$ polymorphism with smoking on the onset of young acute myocardial infarction. Thromb Haemost 2003; 90: $132-139$.

\section{Received 30 December 2003}

Accepted 16 February 2004 


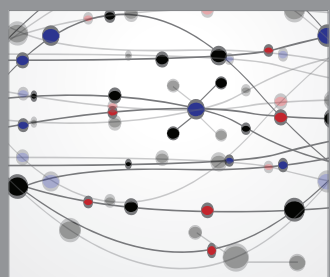

The Scientific World Journal
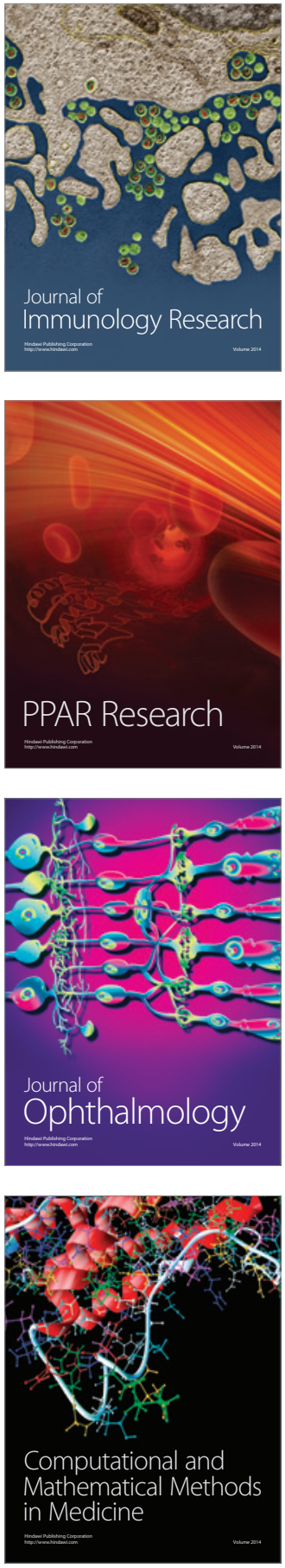

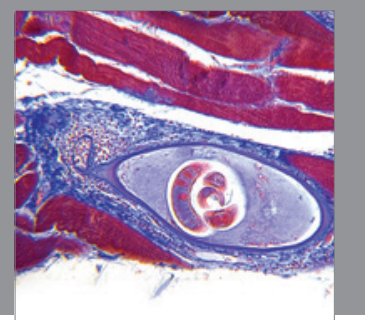

Gastroenterology

Research and Practice
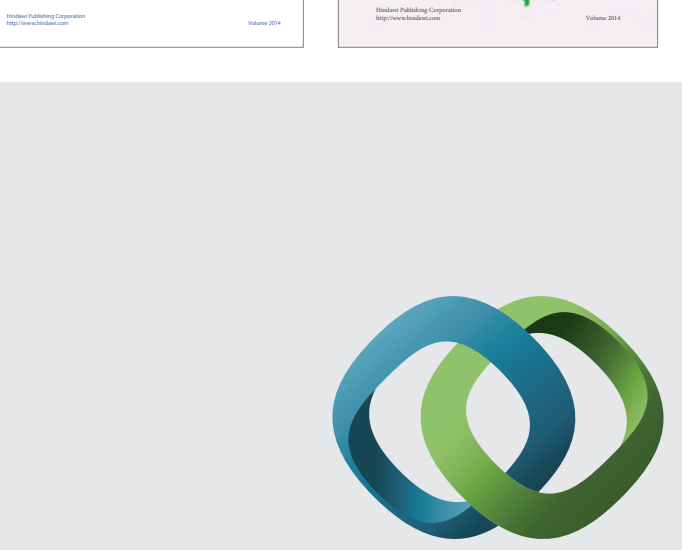

\section{Hindawi}

Submit your manuscripts at

http://www.hindawi.com
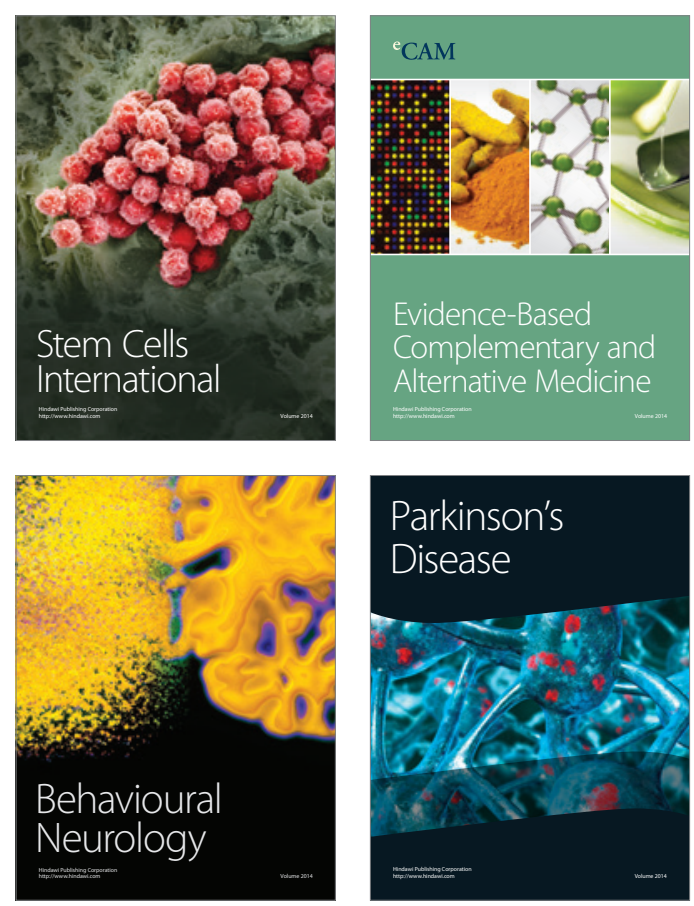

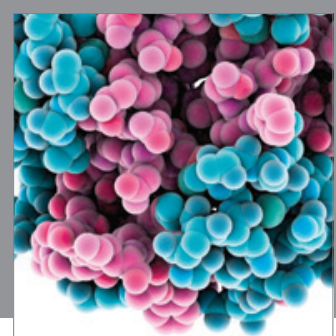

Journal of
Diabetes Research

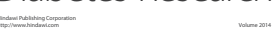

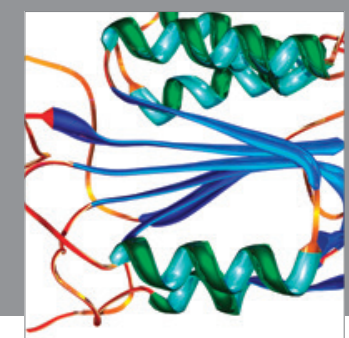

Disease Markers
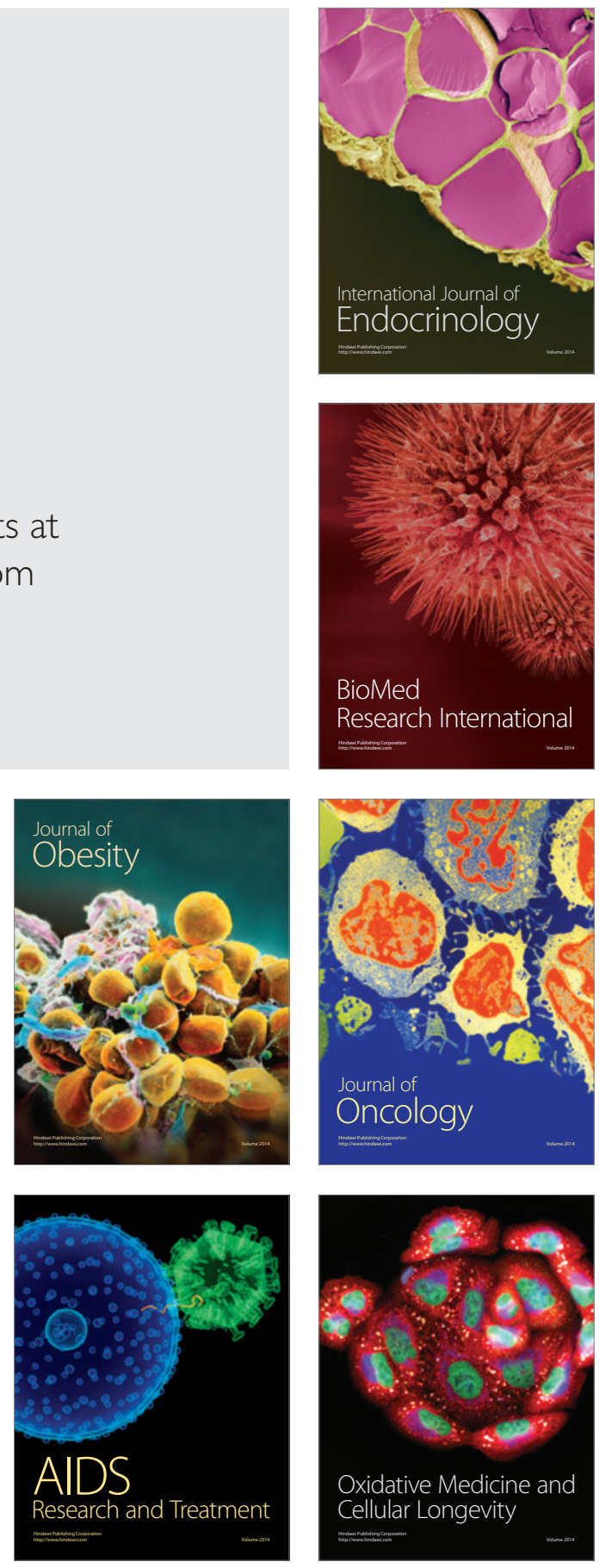